\title{
AN UNATTACHED MASS FOUND IN THE ABDOMINAL CAVITY OF A MALE.
}

\author{
By R. P. Campbeil, M.D., \\ GENITO-URINARY BURGEON, MONTREAL GENERAL HOBPITAK, \\ AND \\ J. J. OWER, M.D., \\ gENIOR AggigTANT PATHOLOGIST, MONTREAL GENERAL hOSPITAL.
}

(From the Genito-urinary Department and the Pathological Laboratory, Montreal General Hospital.)

INASMUCH as the following case report is extraordinary, it is of interest, but insofar as it raises the question of intra-abdominal growth without obvious nourishment, it is perhaps of more scientific importance. It is, therefore, best to leave any discussion until the facts are portrayed.

A. McL., aged sixty-nine years, was admitted to the Montreal General Hospital November 10, 1912. He had suffered from digestive disturbances for forty years, more or less, complaining chiefly of constipation, a burning sensation in the stomach after eating, and of distention and discomfort from gas in the bowels. There was no nausea or vomiting. Two or three years ago he had attacks of pain in the region of the appendix, which called on one occasion for morphin. He did not vomit in these attacks.

For a period of about twenty-five years he can remember a mass in the right side of the abdomen, which at first was quite small, smaller than a hen's egg, and was always freely movable, so movable that it would disappear, apparently into the pelvis, for two or three days at a time. He could push it into any part of the abdomen he wished, but it was first found in the right hypochondrium, and he was most comfortable when it remained in that situation. It always tended to follow the laws of gravity, and in consequence was generally to be found in the most dependent part of the abdomen, falling from side to side as he turned in bed. He had the impression that it was a floating kidney, and indeed he had been told by his medical advisers that this was the most probable diagnosis. The urine, however, had until recently never shown any pathological changes, nor had he suffered any renal crises. He had had pain in the region of the right hypochondrium of a more or less indefinite nature.

We had an opportunity of discussing the case with his physician, who had noted the mass when he first examined him six years previously. It was then the size of a large hen's egg, and of the present consistence. It had, to his certain knowledge, increased 
in size to its present dimensions, which may be described as those of a large orange or a small grapefruit.

The patient came to the Montreal General Hospital on account of an attack of cystitis, with retention, which he had developed in consequence of an hypertrophied prostate gland.

On examination he was found to be a spare, rather pale old man, with little or no subcutaneous fat; arteries moderately sclerosed; normal lungs and heart; rather anemic, and altogether frail. His urine contained considerable pus, and he suffered almost complete retention, due to a moderately hypertrophied prostate gland.

The abdomen was scaphoid, the umbilicus prominent, and the walls soft and non-resistant, making palpation easy. In the right upper quadrant was a hard, slightly irregular mass the size of a large orange. This could be turned about so as to present slightly different aspects to the examining hand, could be pushed downward, and even across the spine to the left side without any trouble, and would stay where placed. It was too large to go down into the pelvis, but this was the only apparent reason why it should not do so, it was so freely movable.

An $x$-ray examination gave no shadow. It was not tender. It was dull on percussion, and was evidently situated in front of the bowels. It was not kidney, but the kidneys were not palpable. The two most marked features about it were its extreme mobility and its marked hardness. The diagnosis of an omental tumor was made.

OPERATION.-The patient was not a good operative risk, owing to the infection of his urinary tract, which apparently involved the kidneys. He was, therefore, put upon catheter life, with daily bladder lavage. So anxious was he, however, that something should be done for the abdominal condition, and so simple did the operation appear, that on November 18, 1912, under gas and oxygen anesthesia, the abdomen was opened directly over the mass, which had to be held stationary by an assistant. In cutting through the peritoneum, we came at once upon the tumor, which showed a pearly white glistening surface, exactly comparable to a uterine fibroid. Pressure on either side of the wound caused the mass to pop out of the wound on to the abdominal wall. It had absolutely no pedicle, nor any attachment whatever. It bore no apparent relation to any viscus, nor was there an adhesion of any kind attached to it. Indeed, on searching the abdomen there did not seem to be any adhesions present. There was, however, a small amount of fluid about the loops of small bowel. The wound was closed, and the patient did well for the next seven or eight days, when he began to fail, with frequent attacks of vomiting and hiccough. The wound, however, remained normal, and it was evident that the lesion was outside the peritoneal cavity. He died January 6, 1913, forty-eight days after the operation. 
The autopsy protocol is as follows:

Montreal General Hospital, 13.8, January 7, 1913. Ten hours postmortem.

The body is that of a poorly developed, much emaciated male adult. No edema is present, but considerable lividity of the dependent parts. Rigor mortis is marked.

Abdomen. There is an elevated bluish scar in the middle line, between the umbilicus and the ensiform cartilage, $13 \mathrm{~cm}$. in length (operation wound).

Peritoneal Cavity. The peritoneal cavity shows no excess of fluid. The peritoneum is smooth and shiny, but darkly pigmented on both parietal and visceral layers. Mesentery is bluish-black. Mesenteric glands are slightly enlarged and darkly pigmented. There are extensive general fibrous adhesions in the abdominal cavity between the liver and colon, the spleen and colon, and binding down the cecum to the posterior abdominal wall. The appendix is retrocecal, surrounded by fibrous adhesions. In one of the fibrous adhesions between the transverse colon and the under surface of the left side of the diaphragm is found a hard calcified mass $0.5 \mathrm{~cm}$. in diameter. It is not attached to the mesentery. A similar mass, perfectly spherical, measuring $0.7 \mathrm{~cm}$. in diameter, is found under the peritoneum of the small bowel about 36 inches above the ileocecal valve. This body is white and calcified, with a smooth surface under glistening peritoneum, and is $2 \mathrm{~cm}$. from the mesenteric attachment (Fig. 1).

Pleural Cavity. This contains no excess of fluid, but there are extensive fibrous adhesions on both sides.

Pericardial cavity. The pericardial cavity contains no excess of body fluid. There are extensive adhesions, delicate and recent, and firm and old between both layers of the pericardium.

Heart. The heart is $320 \mathrm{gm}$. in weight. It is pale brown on section, and the muscle is soft: The endocardium is normal. The aortic arch shows some raised whitish discoloration. The coronary arteries are patent and show a moderate degree of atheromatous change.

Lungs. The right upper and lower lobes are full and firm to the touch. On section they are of a general grayish, mottled appearance. There are many raised gray areas alternating with edematous lung tissue. Even on gentle pressure, much pus and purulent fluid exudes from these areas. The right middle lobe is dark red in color and edematous. The upper and lower lobes are nodular and uneven to the touch. The lower lobe on the left side presents a similar condition to that of the upper and lower lobes on the right side. There are several old pleural scars at the apices of both sides, but no calcareous or caseous nodules are found.

Spleen. Normal.

Stomach. Normal. 
Intestines. Normal.

Pancreas. Normal.

Liver. Weight, $1580 \mathrm{gm}$. It shows on the under surface of the right lobe, retroperitoneally, an area $10 \times 6 \mathrm{~cm}$., which forms one of the confines of the large, perinephritic abscess described below. Otherwise the organ shows nothing abnormal.

Kidneys. The right kidney weighs $300 \mathrm{gm}$. There is a perinephritic abscess cavity connected with the upper pole of the kidney, and extending upward to the liver. This cavity measures $12 \times 6 \times 10 \mathrm{~cm}$., and is filled with a greenish-brown purulent fluid. The right kidney shows extensive abscess condition throughout. The right ureter is thickened, and the mucous membrane is red

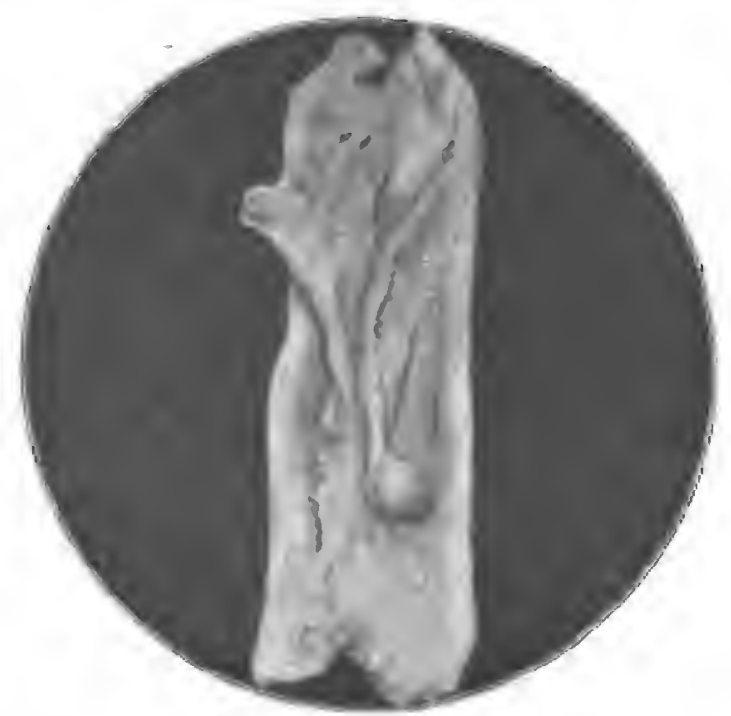

Fia. 1.-Small subperitoneal calcareous nodule found in the small bowel.

and granular. The left kidney is more normal. The cortex averages $0.3 \mathrm{~cm}$. in thickness. The capsule strips with difficulty, and leaves an uneven surface, which shows some retention cysts. The vessels are tortuous. There is no sign of infection.

Right Adrenal. The right adrenal cannot be found, but the left is normal.

Bladder. The bladder is much thickened and trabeculated, with a pigmented and granular mucosa.

Prostate. The prostate is large.

Aorta. The aorta shows marked atheromatous degeneration, especially about the origin of the intercostals. 
CAMPBELL, OWER: UNATTACHED MASS IN ABDOMINAL CAVITY 401

Anatomical Diagnosis. Pyonephrosis; perinephritic abscess (right); acute and chronic pericarditis; bronchopneumonia; chronic cystitis; chronic nephritis; calcified nodules, subperitoneal; hypertrophied prostate.

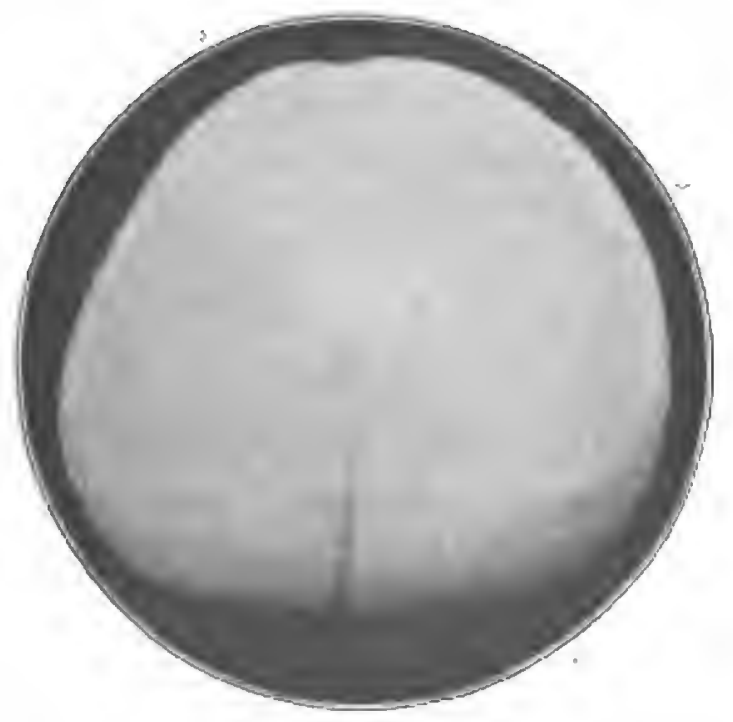

Fia. 2.-View of the mass as removed from the abdominal cavity, about two-thirds actual size.

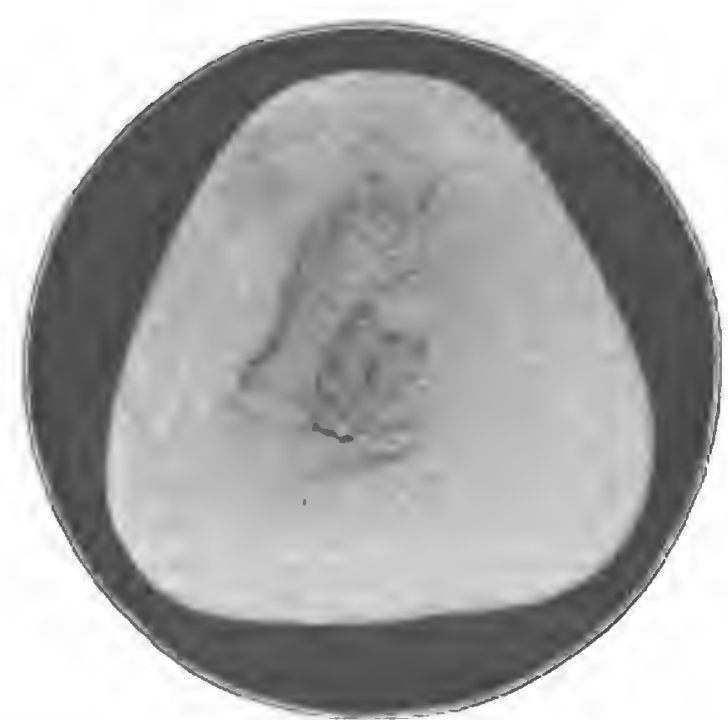

FIG. 3.-View of mass on section, showing calcareous ceatre, but not the concentric arrangement of layers. 
Histological Examination. Montreal General Hospital, S., 12, 635, November 18, 1912.

The tumor is roughly spherical in shape, but with three flattened surfaces or facets (Fig. 2). It measures $9 \times 8 \mathrm{~cm}$. in diameter, and has a smooth, white, glistening surface, without pedicle or any adherent tissue. It is very firm. On section, the mass cuts with some difficulty, and is seen to have a calcareous centre, $1.5 \mathrm{~cm}$. in diameter (Fig. 3). The sectional surface is, for the most part, white and very firm, but an occasional darker ring or lamella accentuates a concentric formation, which is evident in both diameters. The tissue immediately around the calcareous centre is somewhat softer than the remainder.

Sections cut from the mass in various regions show a tissue composed of laminated branching fibers, which take a homogeneous color with methylene blue, and the various differential stains employed. No vessels, cells, nuclei, or fibrils can be demonstrated. In the centre of some fibers, a fine granular deposit is noted, probably calcium salts.

To establish the identity of the tissue is difficult. Were it the remains of a true fibroma, one would expect evidences of vessels, or at least spaces, to persist. The question arises whether it is simply fibrin which has been deposited, layer after layer, on whatever originally was the nucleus, or whether it is collagen remaining from organization of layers of fibrin, similarly deposited by fibroblasts, which have subsequently disappeared, leaving resistant collagen lamellæ.

The tissue takes a homogeneous blue stain with Mallory's connective-tissue stain, and pink with phosphotungstic acid hemotoxylin. These are the reactions for collagen. To try and further rule out fibrin, an attempt was made to digest the tissue with pepsin, controlled by the digestion of fresh fibrin, and formalin fixed fibrin (the tumor tissue having been fixed in formalin), but neither the formalin-fixed fibrin nor the tissue fibers were digested.

Certain facts seem worthy of reiteration:

1. The tumor had been present twenty years or more, and had . been noted by the patient's present physician six years ago.

2. It had always been freely movable.

3. It had never caused pain, other than a feeling of discomfort from moving about.

4. It had grown steadily and slowly from a small nodule, which frequently disappeared for days, to a tumor too large to enter the pelvic inlet.

5. Operation showed it to be absolutely unattached, either by pedicle or by a surrounding envelope of omentum or adhesion.

6 . There is no evidence that a pedicle was ever present.

7. Postmortem examination of the abdomen showed one calcareous nodule on the small bowel, which was, however, entirely 
covered by peritoneum, and another surrounded by adhesions in the neighborhood of the large bowel.

8. The centre of the mass was calcareous.

9. Section of the tumor showed that about this calcareous centre were concentric rings (in three dimensions), similar to those seen about the nucleus of a vesical calculus.

10. Microscopically, the tumor consists of a homogeneous tissue arranged in concentric layers, the exact nature of which cannot be determined, but which probably are either fibrin or organized fibrin, with disappearance of the cellular elements.

As to the origin of the tumor, the following explanations seem worthy of discussion.

1. The tumor may represent a pediculated fibroid or fibroma, which was originally attached to some viscus, or to the abdominal parietes, and became detached, calcified in the centre, and remained a foreign body in the peritoneal cavity. The entire absence of any sign of pedicle, not only on the surface, but in the outline of the concentric rings, vitiates this hypothesis.

2. A calcareous mass similar to those found at autopsy, or indeed a small fibroma in such a situation, might have gradually grown by having new layers added to it from the surrounding envelope of peritoneum, until reaching a certain definite size, its mere weight, or accident tears a way through the peritoneal covering, leaving it a free body in the abdominal cavity.

3. The small tumor or nodule, freed from its attachment in the above manner, may have continued to grow within the abdominal cavity, much as a pearl does in the shell of an oyster, causing a certain irritation of the peritoneum, and an outpouring of fluid and cells (there was definite fluid present). The coagulation of fibrin over the offending surface and the gradual organization of the same might follow. The definitely recurring layers are in favor of some such view.

4. A combination of the second and third is, of course, another possibility, and is perhaps the most likely. That is to say, the earliest growth took place subperitoneally or elsewhere, and the later growth, while it was a free body in the peritoneal cavity. Calcification may have occurred at any time.

We have been unable to find any report of a similar case, though it is quite possible that such may have escaped our notice, owing to the difficulty of classifying an unusual condition in the literature.

Fibromas of the abdominal parietes and contents have been noted, but are not common. The instances recorded do not seem to throw any light upon the present case. 